\title{
Fibonacci Numbers with the Lehmer Property
}

by

\author{
Florian LUCA
}

\author{
Presented by Andrzej SCHINZEL
}

Summary. We show that if $m>1$ is a Fibonacci number such that $\phi(m) \mid m-1$, where $\phi$ is the Euler function, then $m$ is prime.

Let $\phi(n)$ be the Euler function of the positive integer $n$. Clearly, $\phi(n)=$ $n-1$ if $n$ is a prime. Lehmer [9] (see also B37 in [7]) conjectured that if $\phi(n) \mid n-1$, then $n$ is prime. To this day, no counterexample to this conjecture (and no proof of it either) has been found. Let us say that $n$ has the Lehmer property if $n$ is composite and $\phi(n) \mid n-1$. Thus, Lehmer's conjecture is that there is no number with the Lehmer property.

Pomerance (see [14], [15]) showed that if $\mathcal{L}(x)$ denotes the number of numbers $n \leq x$ with the Lehmer property then the estimate

$$
\mathcal{L}(x)=O\left(x^{1 / 2}(\log x)^{3 / 4}(\log \log x)^{-1 / 2}\right)
$$

holds, where $\log x$ stands for the natural logarithm of $x$. The exponent $3 / 4$ of $\log x$ in the above bound was successively lowered to $1 / 2$ by Zhun [18] and to 0 (at the cost of some extra power of $\log \log x$ ) by Banks and Luca [2].

In the recent paper [6], Diaconescu studied numbers with the Lehmer property and some extra structure and concluded that there should be only finitely many of them. For example, he showed that if $k \geq 1$ is a fixed positive integer then there are only finitely many positive integers $n$ with the Lehmer property which also satisfy the congruence $\phi(n)^{k} \equiv 1(\bmod n)$.

Here, we study the numbers with the Lehmer property which belong to a familiar subset of positive integers, namely the Fibonacci numbers. Recall

2000 Mathematics Subject Classification: 11A20, 11B39.

Key words and phrases: Fibonacci number, Lucas number, Euler function, Lehmer's conjecture. 
that the sequence of Fibonacci numbers $\left(F_{n}\right)_{n \geq 0}$ has $F_{0}=0, F_{1}=1$ and $F_{n+2}=F_{n+1}+F_{n}$ for all $n \geq 0$. Our result is the following.

THEOREM 1. There is no Fibonacci number with the Lehmer property.

Throughout this paper, we use $p$ with or without subscripts for a prime number. For a positive integer $m$ we write $\omega(m)$ and $\tau(m)$ for the number of distinct prime divisors of $m$ and the total number of positive integer divisors of $m$, respectively. Recall that if $m=p_{1}^{\alpha_{1}} \cdots p_{k}^{\alpha_{k}}$, where $p_{1}, \ldots, p_{k}$ are distinct primes and $\alpha_{1}, \ldots, \alpha_{k}$ are positive integer exponents, then $\omega(m)=k$ and $\tau(m)=\left(\alpha_{1}+1\right) \cdots\left(\alpha_{k}+1\right)$.

We also recall that if we write $\alpha=(1+\sqrt{5}) / 2$ and $\beta=(1-\sqrt{5}) / 2$, then $F_{n}=\left(\alpha^{n}-\beta^{n}\right) /(\alpha-\beta)$ for all $n \geq 0$. This is sometimes called the Binet formula. Furthermore, if we write $\left(L_{n}\right)_{n \geq 0}$ for the Lucas sequence given by $L_{0}=2, L_{1}=1$ and $L_{n+2}=L_{n+1}+L_{n}$ for all $n \geq 0$, then both the Binet formula $L_{n}=\alpha^{n}+\beta^{n}$ and

$$
L_{n}^{2}-5 F_{n}^{2}=4(-1)^{n}
$$

hold for all $n \geq 0$.

Acknowledgements. The author thanks the referee for a careful reading of the manuscript and Professors Janitzio Mejía Huguet, Alvaro Alvarez Parilla and Mihai Stoiciu for useful conversations. Work on this paper started during an enjoyable visit at the Mathematics Department of the Universidad Autónoma de Baja California in Ensenada, Mexico in November of 2006. The author thanks the people there for their warm hospitality.

1. The proof. Assume that $n>2$ and that $F_{n}$ is a composite positive integer such that $\phi\left(F_{n}\right) \mid F_{n}-1$. Lehmer [9] showed that $\omega\left(F_{n}\right) \geq 7$ and this was subsequently improved to 11 by Lieuwens [10], to 13 by Kishore [8], and to 14 by Cohen and Hagis [5]. When $3 \mid F_{n}$, Lieuwens [10] showed that in fact $F_{n}>5.5 \cdot 10^{570}$. Since certainly $\phi\left(F_{n}\right)$ is even, we infer that $F_{n}$ is odd. Thus, either $3 \mid F_{n}$ and $F_{n} \geq 5.5 \cdot 10^{570}$, or

$$
F_{n} \geq 5 \cdot 7 \cdot 11 \cdot 13 \cdots 53 \text {. }
$$

In both cases, we see that $n \geq 50$. Let $K=\omega\left(F_{n}\right)$. By Theorem 4 in [15], we have $F_{n}<K^{2^{K}}$. It is easy to check by induction that $F_{s}>2^{s / 2}$ for all $s>10$. Since $n>50$, we have $K^{2^{K}}>F_{n}>2^{n / 2}$, therefore

$$
2^{K} \log K>\frac{n \log 2}{2}>\frac{n}{3} \text {. }
$$

We now check that the above inequality (2) implies that

$$
2^{K}>\frac{n}{4 \log \log n} \text {. }
$$


Indeed, assume that the reverse inequality

$$
2^{K} \leq \frac{n}{4 \log \log n}
$$

holds. Then

$$
K \log 2<\log n-\log 4-\log \log \log n<\log n .
$$

In the rightmost inequality above we used the fact that $n>50>e^{e}$, so $\log \log n>1$, therefore $\log \log \log n$ is positive. Thus, $K<(\log n) / \log 2<$ $2 \log n$, therefore

$$
2^{K} \log K<\frac{n \log (2 \log n)}{4 \log \log n}=\frac{n}{4}+\frac{\log 2}{4 \log \log n} .
$$

Comparing the last inequality above with (2), we get

$$
\frac{n}{3}<\frac{n}{4}+\frac{\log 2}{4 \log \log n}
$$

therefore

$$
n<\frac{3 \log 2}{\log \log n}<\frac{3 \log 2}{\log \log 50}<2,
$$

which is impossible. Thus, inequality (2) holds.

In what follows, we will use the following well-known relations (see, for example, Lemma 2 in [11]):

$$
\begin{aligned}
& F_{4 m}-1=F_{2 m+1} L_{2 m-1}, \quad F_{4 m+1}-1=F_{2 m} L_{2 m+1}, \\
& F_{4 m+2}-1=F_{2 m} L_{2 m+2}, \quad F_{4 m+3}-1=F_{2 m+2} L_{2 m+1},
\end{aligned}
$$

which can be easily verified using the Binet formulae.

We split the remaining analysis in two cases.

CASE 1: $n$ is odd. Let $p$ be any prime factor of $F_{n}$. Clearly, $p$ is odd. Reducing relation $(1)$ modulo $p$ we get $L_{n}^{2} \equiv-4(\bmod p)$, so we infer that -1 is a quadratic residue modulo $p$. In particular, $p \equiv 1(\bmod 4)$. Since this is true for all prime factors $p$ of $F_{n}$, we conclude that $2^{2 K} \mid \phi\left(F_{n}\right)$. Since $n=2 m+1$ is odd, formulae (4) tell us that $F_{n}-1=F_{(n-1) / 2} L_{(n+1) / 2}$ or $F_{(n+1) / 2} L_{(n-1) / 2}$ according as $m$ is even or odd. Thus, we get

$$
2^{2 K}\left|\phi\left(F_{n}\right)\right| F_{n}-1 \mid F_{(n-\varepsilon) / 2} L_{(n+\varepsilon) / 2} \quad \text { for some } \varepsilon \in\{ \pm 1\} \text {. }
$$

The period of the sequence $\left(L_{s}\right)_{s \geq 0}$ modulo 8 is 12 . Furthermore, listing the first twelve members of $\left(L_{s}\right)_{s \geq 0}$ one notices that none of them is a multiple of 8 . Thus, the above divisibility condition certainly implies that $2^{2 K-2}$ divides either $F_{(n-1) / 2}$ or $F_{(n+1) / 2}$. It is well-known and easy to check by induction that if $\ell \geq 3$ and $2^{\ell} \mid F_{s}$, then $2^{\ell-2} \cdot 3 \mid s$. Since $2 K-2 \geq 2 \cdot 14-2>3$, we find that $2^{2 K-4} \cdot 3$ divides one of $(n-1) / 2$ or $(n+1) / 2$. Thus, using also 
inequality (3), we have

$$
\frac{n+1}{2} \geq 2^{2 K-4} \cdot 3 \geq \frac{3}{16}\left(\frac{n}{4 \log \log n}\right)^{2},
$$

therefore

$$
n^{2}<\frac{128}{3}(n+1)(\log \log n)^{2}
$$

leading to $n<101$.

CAse 2: $n$ is even. Here, we write $n=2 m$, so $F_{n}=F_{2 m}=F_{m} L_{m}$. Relation (1) together with the fact that $F_{n}$ is odd implies that $F_{m}$ and $L_{m}$ are coprime, so $\phi\left(F_{n}\right)=\phi\left(F_{m} L_{m}\right)=\phi\left(F_{m}\right) \phi\left(L_{m}\right)$. Let $m=p_{1}^{\alpha_{1}} \cdots p_{k}^{\alpha_{k}}$ be the factorization of $n$, where $p_{1}<\cdots<p_{k}$ are distinct primes. We shall spend a lot of time bounding $p_{1}$.

We start by noticing that $m$ has to be odd (so $p_{1}>2$ ). Indeed, assume that $m=2 m_{0}$ is even. Then formula (4) tells us that $\phi\left(F_{n}\right) \mid F_{2 m_{0}+1} L_{2 m_{0}-1}$. As we have said before, 8 cannot divide $L_{s}$ for any value of the positive integer $s$. Furthermore, if $8 \mid F_{s}$, then $6 \mid s$, and in particular $s$ is even. Since $2 m_{0}+1$ is odd, we conclude that $F_{2 m_{0}+1}$ is not a multiple of 8 . Thus, 32 cannot divide $F_{2 m_{0}+1} L_{2 m_{0}-1}$, but this is impossible since $2^{K} \mid \phi\left(F_{n}\right)$ and $K \geq 14$. Hence, $m$ is odd, therefore $p_{1}>2$. If $p_{1}=3$, then $F_{n}$ is even, which is not the case. Thus, $p_{1} \geq 5$.

By the primitive divisor theorem for the Fibonacci and Lucas numbers (see [4]), for each divisor $d>1$ of $m$ there exists a prime $p \mid L_{d}$ such that $p \nmid L_{d_{1}}$ for all $0<d_{1}<d$. Since $m$ is odd, Binet's formula implies that $p \mid L_{m}$. Reducing relation (1) modulo $p$, we get $-5 F_{m}^{2} \equiv-4(\bmod p)$, therefore $5 F_{m}^{2} \equiv 4(\bmod p)$. This shows that 5 is a quadratic residue modulo $p$, so by quadratic reciprocity, $p$ is a quadratic residue modulo 5 also. Thus, $p \equiv 1$ $(\bmod d)$, therefore $d \mid p-1$. Now let $d$ be an arbitrary divisor of $m$ which is a multiple of $p_{1}$. The number of such divisors is at least $\tau\left(m / p_{1}\right)$. For each such $d$, there is a primitive prime factor $p_{d}$ of $L_{d}$ such that $p_{1}|d|$ $p_{d}-1\left|\phi\left(L_{d}\right)\right| \phi\left(L_{m}\right)$. This shows that the exponent $\ell_{1}$ of $p_{1}$ in $\phi\left(L_{m}\right)$ is at least $\tau\left(m / p_{1}\right)$. Thus,

$$
p_{1}^{\tau\left(m / p_{1}\right)}\left|p_{1}^{\ell_{1}}\right| \phi\left(L_{m}\right)\left|\phi\left(L_{n}\right)\right| F_{2 m}-1 \mid F_{m-1} L_{m+1}
$$

and $p_{1} \mid m$. Let $z\left(p_{1}\right)$ be the order of appearance of $p_{1}$ in the Fibonacci sequence, i.e., the smallest positive integer $s$ such that $p_{1} \mid F_{s}$. It is known that $z\left(p_{1}\right) \mid p_{1}-e$, where $e$ is the Legendre symbol $\left(5 / p_{1}\right)$; hence, it is 1 if $p_{1} \equiv \pm 1(\bmod 5)$, it is -1 if $p_{1} \equiv \pm 2(\bmod 5)$, and it is 0 if $p_{1}=5$. Let $a_{1}$ be the exponent of $p_{1}$ in $F_{z\left(p_{1}\right)}$. Since $p_{1} \mid F_{m-1} L_{m+1}$, we find that either $p_{1} \mid F_{m-1}$ or $p_{1} \mid L_{m+1}$. Since $L_{m+1} \mid F_{2(m+1)}$, we further deduce that either $p_{1} \mid F_{m-1}$ or $p_{1} \mid F_{2(m+1)}$. Let us notice that $p_{1}$ can divide only one 
but not both of the above numbers. Indeed, since $F_{m-1} \mid F_{2(m-1)}$, it follows that if $p_{1}$ divides both the above numbers, then it divides both $F_{2 m-2}$ and $F_{2 m+2}$. But then $p_{1} \mid F_{\text {gcd }(2 m-2,2 m+2)}$, and $\operatorname{gcd}(2 m-2,2 m+2) \mid 4$. However, $F_{4}=3$ and we have already seen that $p_{1}>3$. Thus, only one of $m-1$ or $2(m+1)$ is divisible by $z\left(p_{1}\right)$, therefore $p_{1}^{a_{1}}$ divides either $F_{m-1}$ or $F_{2(m+1)}$. It is well-known that if $\ell>a_{1}$ and $p_{1}^{\ell} \mid F_{s}$, then $p_{1} z\left(p_{1}\right) \mid s$. Since $p_{1} \mid m$ and $p_{1}$ is odd, it follows that $p_{1} z\left(p_{1}\right)$ can divide neither $m-1$ nor $2 m+2$. The conclusion is that $\ell_{1} \leq a_{1}$, therefore $p_{1}^{\ell_{1}} \leq p_{1}^{a_{1}}$. In particular, $\ell_{1}=a_{1}=1$ if $p_{1}=5$ or $p_{1}=7$.

Assume now that $p_{1} \geq 11$. Then $p_{1}^{a_{1}} \mid F_{p_{1}-e}=F_{\left(p_{1}-e\right) / 2} L_{\left(p_{1}-e\right) / 2}$. The greatest common divisor of $F_{\left(p_{1}-e\right) / 2}$ and $L_{\left(p_{1}-e\right) / 2}$ is at most 2 (by (1) for $\left.n=\left(p_{1}-e\right) / 2\right)$ and $p_{1}$ is odd, so either $p_{1}^{a_{1}} \mid F_{\left(p_{1}-e\right) / 2}$ or $p_{1}^{a_{1}} \mid L_{\left(p_{1}-e\right) / 2}$. Since $F_{s}<\alpha^{s}$ for all positive integers $s$, as can be easily verified by induction, we see that when $p_{1}^{a_{1}} \mid F_{\left(p_{1}-e\right) / 2}$, we have

$$
p_{1}^{a_{1}} \leq F_{\left(p_{1}-e\right) / 2} \leq F_{\left(p_{1}+1\right) / 2} \leq \alpha^{\left(p_{1}+1\right) / 2},
$$

so

$$
\tau\left(m / p_{1}\right) \leq \ell_{1} \leq a_{1} \leq \frac{\left(p_{1}+1\right) \log \alpha}{2 \log p_{1}} .
$$

The same conclusion, namely that $p_{1}^{a_{1}}<\alpha^{\left(p_{1}+1\right) / 2}$, is also reached when $p_{1}^{a_{1}} \mid L_{\left(p_{1}-e\right) / 2}$, in the following way. First observe that the above inequality is certainly true when $a_{1}=1$ since $p_{1} \geq 11$. Now assume that $a_{1}>1$. If $L_{\left(p_{1}-e\right) / 2}=p_{1}^{a_{1}}$, then, in particular, $L_{\left(p_{1}-e\right) / 2}$ is a perfect power. However, by the recent results from [3], there is no perfect power of the form $L_{s}$ for $s>3$. Hence,

$$
p_{1}^{a_{1}} \leq \frac{1}{2} L_{\left(p_{1}-e\right) / 2}<\frac{1}{2}\left(\alpha^{\left(p_{1}-e\right) / 2}+1\right)<\alpha^{\left(p_{1}+1\right) / 2},
$$

which implies inequality (6). Now note that

$$
\tau\left(m / p_{1}\right)=\alpha_{1}\left(\alpha_{2}+1\right) \cdots\left(\alpha_{k}+1\right) \geq\left(\frac{\alpha_{1}+1}{2}\right)\left(\alpha_{2}+1\right) \cdots\left(\alpha_{k}+1\right)=\frac{\tau(m)}{2},
$$

therefore

$$
\tau(m) \leq 2 \tau\left(m / p_{1}\right) \leq \frac{\left(p_{1}+1\right) \log \alpha}{\log p_{1}} .
$$

Now observe that $\phi\left(F_{n}\right) \mid F_{n}-1$ and $\phi\left(F_{n}\right)<F_{n}-1$. Thus, $F_{n}-1 \geq 2 \phi\left(F_{n}\right)$, therefore

so

$$
2 \leq \frac{F_{n}}{\phi\left(F_{n}\right)} \leq \prod_{p \mid F_{n}}\left(1+\frac{1}{p-1}\right)<\exp \left(\sum_{p \mid F_{n}} \frac{1}{p-1}\right),
$$

$$
\log 2 \leq \sum_{p \mid F_{n}} \frac{1}{p-1} .
$$


In what follows, we shall exploit the above relation. Since our ultimate goal is to bound $p_{1}$, we shall from now on assume that $p_{1}>1000$.

Let us now take a closer look at the right hand side of inequality (8). For each divisor $d>1$ of $m$, let $\mathcal{P}_{d}$ be the set of primitive prime factors of $F_{2 d}=F_{d} L_{d}$. All these primes are $\equiv \pm 1(\bmod d)$ and are odd. In particular, the smallest one is $\geq 2 d-1$. Assume that $\ell_{d}=\# \mathcal{P}_{d}$ is their number. Then

$$
(2 d-1)^{\ell_{d}} \leq F_{2 d}<\alpha^{2 d},
$$

so

$$
\ell_{d}<\frac{2 d \log \alpha}{\log (2 d-1)} .
$$

We next show that the estimate

$$
\sum_{p \in \mathcal{P}_{d}} \frac{1}{p-1} \leq \frac{1.8}{d}+\frac{4.3 \log \log d}{d}
$$

holds for our ranges of variables. Observe that

$$
\begin{aligned}
\sum_{p \in \mathcal{P}_{d}} \frac{1}{p-1} & =\sum_{p \in \mathcal{P}_{d}} \frac{1}{p}+\sum_{p \in \mathcal{P}_{d}} \frac{1}{p(p-1)} \leq \sum_{p \in \mathcal{P}_{d}} \frac{1}{p}+\frac{\ell_{d}}{(2 d-2)(2 d-1)} \\
& \leq \frac{1}{2 d-1}+\frac{1}{2 d+1}+\sum_{3 d<p<d^{2}} \frac{1}{p}+\ell_{d}\left(\frac{1}{d^{2}}+\frac{1}{(2 d-2)(2 d-1)}\right) .
\end{aligned}
$$

For coprime integers $a$ and $b$ and a positive real number $t$ let $\pi(t ; a, b)$ be the number of primes $p \leq t$ with $p \equiv a(\bmod b)$. The large sieve inequality of Montgomery and Vaughan [13] tells us that

$$
\pi(t ; a, b) \leq \frac{2 t}{\phi(b) \log (t / b)}
$$

for all $t>b$ and all $a$ coprime to $b$. Since the set of primes $p \in\left(3 d, d^{2}\right)$ which belong to $\mathcal{P}_{d}$ is contained in the set of primes $p \equiv \pm 1(\bmod d)$, it follows, by Abel's summation formula, that

$$
\begin{aligned}
\sum_{3 d<p<d^{2}} \frac{1}{p} & \leq \sum_{\substack{3 d<p \leq d^{2} \\
p \equiv-1(\bmod d)}} \frac{1}{p}+\sum_{\substack{3 d<p \leq d^{2} \\
p \equiv 1(\bmod d)}} \frac{1}{p} \\
& \leq \frac{\pi\left(d^{2} ;-1, d\right)+\pi\left(d^{2} ; 1, d\right)}{d^{2}}+\int_{3 d}^{d^{2}} \frac{\pi(t ;-1, d)+\pi(t ; 1, d)}{t^{2}} d t \\
& \leq \frac{4 d^{2}}{\phi(d) d^{2} \log \left(d^{2} / d\right)}+\frac{4}{\phi(d)} \int_{3 d}^{d^{2}} \frac{d t}{t \log (t / d)} \\
& =\frac{4}{\phi(d) \log d}+\left.\frac{4}{\phi(d)} \log \log (t / d)\right|_{t=3 d} ^{t=d^{2}} \\
& <\frac{4}{\phi(d) \log d}+\frac{4 \log \log d}{\phi(d)}
\end{aligned}
$$


because $\log \log 3>0$. As for $\phi(d)$ versus $d$, note that, by inequality (7),

$$
\begin{aligned}
\frac{d}{\phi(d)} & \leq \prod_{p \mid m}\left(1+\frac{1}{p-1}\right) \leq\left(1+\frac{1}{p_{1}-1}\right)^{\tau(m)} \\
& \leq \exp \left(\frac{\tau(m)}{p_{1}-1}\right) \leq \exp \left(\frac{(\log \alpha)\left(p_{1}+1\right)}{\left(p_{1}-1\right) \log p_{1}}\right)<1.073
\end{aligned}
$$

because $p_{1}>10^{3}$. Thus, $d / \phi(d) \leq 1.073$, so putting all of the above estimates together we get

$$
\begin{aligned}
\sum_{p \in \mathcal{P}_{d}} \frac{1}{p-1} \leq & \frac{1}{2 d-1}+\frac{1}{2 d+1}+\ell_{d}\left(\frac{1}{d^{2}}+\frac{1}{(2 d-2)(2 d-1)}\right) \\
& +\frac{4.3}{d \log d}+\frac{4.3 \log \log d}{d} .
\end{aligned}
$$

Since $d \geq p_{1}>10^{3}$, we have

$$
\begin{aligned}
& \frac{1}{2 d-1}+\frac{1}{2 d+1}+\ell_{d}\left(\frac{1}{d^{2}}+\frac{1}{(2 d-2)(2 d-1)}\right)+\frac{4.3}{d \log d} \\
& \leq \frac{1}{d}\left(\frac{4 \cdot 10^{6}}{4 \cdot 10^{6}-1}+\frac{2 \log \alpha}{\log \left(2 \cdot 10^{3}-1\right)}\left(1+\frac{1}{\left(2-2 / 10^{3}\right)\left(2-1 / 10^{3}\right)}\right)+\frac{4.3}{\log \left(10^{3}\right)}\right) \\
& <\frac{1.8}{d}
\end{aligned}
$$

which together with inequality (10) gives

$$
\sum_{d \in \mathcal{P}_{d}} \frac{1}{p-1}<\frac{1.8}{d}+\frac{4.3 \log \log d}{d}
$$

which is the promised inequality (9).

Since the function $x \mapsto(\log \log x) / x$ is decreasing for $x>10$, we have

$$
\begin{aligned}
\sum_{p \mid F_{n}} \frac{1}{p-1} & =\sum_{d \mid m} \sum_{p \in \mathcal{P}_{d}} \frac{1}{p-1} \leq \sum_{d \mid m, d>1}\left(\frac{1.8}{d}+\frac{4.3 \log \log d}{d}\right) \\
& \leq\left(\frac{1.8}{p_{1}}+\frac{4.3 \log \log p_{1}}{p_{1}}\right) \tau(m) \\
& \leq(\log \alpha) \frac{\left(p_{1}+1\right)}{\log p_{1}} \cdot\left(\frac{1.8}{p_{1}}+\frac{4.3 \log \log p_{1}}{p_{1}}\right),
\end{aligned}
$$

and comparing (11) with (8), we get

$$
\log p_{1} \leq \frac{\log \alpha}{\log 2} \frac{p_{1}+1}{p_{1}}\left(1.8+4.3 \log \log p_{1}\right) .
$$


Since $p_{1}>10^{3}$, we get

$$
\frac{\log \alpha}{\log 2}\left(1+\frac{1}{p_{1}}\right)<0.7
$$

Hence, with $x=\log p_{1}$, we get $x<0.7(1.8+4.3 \log x)$, which implies that $x<7.21$, therefore $p_{1}=e^{x}<e^{7.21}<1400$. Thus, $p_{1}<1400$. We have finally bounded $p_{1}$.

At this point, we recall that D. D. Wall [17] conjectured that $p \| F_{z(p)}$ for all primes $p$. No counterexample to this conjecture (nor a proof of it either) has been found. Sun and Sun [16] deduced that the so-called first case of Fermat's Last Theorem is impossible under Wall's conjecture. We checked with Mathematica that Wall's conjecture is true for all $p<1400$. In fact, in [1] it is mentioned that recently McIntosh and Roettger [12] verified Wall's conjecture for all $p<10^{14}$ and found it to be true. In particular, it is true for $p_{1}$. This shows that $a_{1}=1$ for all possible values of $p_{1}$, therefore $\tau\left(m / p_{1}\right)=1$, so $m=p_{1}$ and $L_{p_{1}}$ is a prime. But in this case, $F_{m}=F_{p_{1}}$ has $K-1$ prime factors and $m$ is odd, so by the arguments from Case 1 each prime factor of $F_{m}$ is congruent to 1 modulo 4 . Thus, $2^{2 K-1}\left|\phi\left(F_{n}\right)\right| F_{m-1} L_{m+1}$. Since 8 cannot divide $L_{m+1}$, we infer that $2^{2 K-3} \mid F_{m-1}$, therefore $2^{2 K-5} \cdot 3 \mid m-1$. We thus find, using inequality (3), that

$$
\frac{n}{2}>\frac{n}{2}-1=m-1 \geq 2^{2 K-5} \cdot 3 \geq \frac{3}{32}\left(\frac{n}{4 \log \log n}\right)^{2},
$$

therefore

$$
n<\frac{256}{3}(\log \log n)^{2},
$$

leading to $n<250$.

Thus, in both cases of $n$ odd or $n$ even we arrived at the conclusion that $n<250$. We now checked that there is no Fibonacci number $F_{n}$ with $n<250$ having the Lehmer property in the following way. We used Mathematica to show that if $\omega\left(F_{n}\right) \geq 14$ and $n<250$, then $n \in\{180,210,240\}$. Then we used again Mathematica and checked that for these three values of $n$, the ratio $\left(F_{n}-1\right) / \phi\left(F_{n}\right)$ is not an integer.

This completes the proof of Theorem 1 .

\section{References}

[1] V. Andrejić, On Fibonacci powers, Univ. Beograd Publ. Elektrotehn. Fak. Sec. Mat. 17 (2006), 38-44.

[2] W. D. Banks and F. Luca, Composite integers $n$ for which $\phi(n) \mid n-1$, Acta Math. Sinica, to appear. 
[3] Y. Bugeaud, M. Mignotte and S. Siksek, Classical and modular approaches to exponential Diophantine equations. I. Fibonacci and Lucas perfect powers, Ann. of Math. (2) 163 (2006), 969-1018.

[4] R. D. Carmichael, On the numerical factors of the arithmetic forms $\alpha^{n} \pm \beta^{n}$, ibid. 15 (1913), 30-70.

[5] G. L. Cohen and P. Hagis, On the number of prime factors of $n$ if $\phi(n) \mid n-1$, Nieuw Arch. Wisk. (3) 28 (1980), 177-185.

[6] M. Diaconescu, On the equation $m-1=a \phi(m)$, Integers 6 (2006), art. 6.

[7] R. K. Guy, Unsolved Problems in Number Theory, Springer, 2004.

[8] M. Kishore, On the equation $k \phi(M)=M-1$, Nieuw Arch. Wisk. (3) 25 (1977), $48-53$.

[9] D. H. Lehmer, On Euler's totient function, Bull. Amer. Math. Soc. 38 (1932), 745751.

[10] E. Lieuwens, Do there exist composite numbers for which $k \phi(M)=M-1$ holds?, Nieuw Arch. Wisk. (3) 18 (1970), 165-169.

[11] F. Luca and L. Szalay, Fibonacci numbers of the form $p^{a} \pm p^{b}+1$, Fibonacci Quart., to appear.

[12] R. J. McIntosh and E. L. Roettger, A search for Fibonacci-Wiefrich and Wolsenholme primes, preprint, 2006.

[13] H. L. Montgomery and R. C. Vaughan, The large sieve, Mathematika 20 (1973), $119-134$.

[14] C. Pomerance, On composite $n$ for which $\phi(n) \mid n-1$, Acta Arith. 28 (1976), 387-389.

[15] -, On composite $n$ for which $\phi(n) \mid n-1$, II, Pacific J. Math. 69 (1977), 177-186.

[16] Z. H. Sun and Z. W. Sun, Fibonacci numbers and Fermat's last theorem, Acta Arith. 60 (1992), 371-388.

[17] D. D. Wall, Fibonacci series modulo m, Amer. Math. Monthly 67 (1960), 525-532.

[18] S. Zun, On composite $n$ for which $\phi(n) \mid n-1$, J. China Univ. Sci. Tech. 15 (1985), 109-112.

Florian Luca

Instituto de Matemáticas

Universidad Nacional Autónoma de México

Ap. Postal 61-3 (Xangari)

C.P. 58089, Morelia, Michoacán, México

E-mail: fluca@matmor.unam.mx

Received December 18, 2006;

received in final form February 20, 2007 